\title{
Decentralized Energy Management of Power Networks with Distributed Generation using Periodical Self-Sufficient Repartitioning Approach
}

\author{
Wicak Ananduta and Carlos Ocampo-Martinez
}

\begin{abstract}
In this paper, we propose a decentralized model predictive control (MPC) method as the energy management strategy for a large-scale electrical power network with distributed generation and storage units. The main idea of the method is to periodically repartition the electrical power network into a group of self-sufficient interconnected microgrids. In this regard, a distributed graph-based partitioning algorithm is proposed. Having a group of self-sufficient microgrids allows the decomposition of the centralized dynamic economic dispatch problem into local economic dispatch problems for the microgrids. In the overall scheme, each microgrid must cooperate with its neighbors to perform repartitioning periodically and solve a decentralized MPC-based optimization problem at each time instant. In comparison to the approaches based on distributed optimization, the proposed scheme requires less intensive communication since the microgrids do not need to communicate at each time instant, at the cost of suboptimality of the solutions. The performance of the proposed scheme is shown by means of numerical simulations with a well-known benchmark case.
\end{abstract}

Index Terms-Economic dispatch, online periodical partitioning, decentralized MPC

\section{INTRODUCTION}

In this paper, we discuss an economic dispatch problem of a power network that consists of multiple distributed generation units, which can be either dispatchable or nondispatchable, such as solar-powered or wind-powered units. Furthermore, storage devices are also introduced into the network as a way to deal with the intermittency issue of the non-dispatchable renewable power generation. A noncentralized scheme based on model predictive control (MPC) is then developed to solve the dispatch problem. MPC framework, with the receding horizon principle, is considered in order to deal with the dynamics of the storage units that must be taken into account as well as with the uncertainties of the loads and non-dispatchable energy generation units.

The most widely recognized approach to design a noncentralized MPC-based strategy for this problem is by implementing a distributed optimization algorithm, such as dual decomposition [1], alternating direction method of multipliers (ADMM) [2], optimal condition decomposition (OCD) [3], and consensus-based algorithms, e.g., [4]. The main advantages of the aforementioned approaches are the ability

W. Ananduta and C. Ocampo-Martinez are with the Institut de Ròbotica i Informàtica Industrial, CSIC-UPC, Carrer Llorens i Artigas 4-6, 08028 Barcelona, Spain (emails: \{wananduta, cocampo\}eiri.upc.edu).

This work has received funding from the European Union's Horizon 2020 research and innovation programme under the Marie Skłodowska-Curie grant agreement No 675318 (INCITE). This work has been also supported by the Spanish State Research Agency through the María de Maeztu Seal of Excellence to IRI (MDM-2016-0656). to handle couplings and the optimality of the solutions when the problem is convex and it is provided that some other mild assumptions also hold. However, the previously mentioned distributed algorithms are iterative and require high flow of exchanged information.

Therefore, in this paper, we propose an alternative decentralized control scheme to deal with the economic dispatch problem. In the proposed scheme, the network is partitioned into a set of microgrids and the dispatch problem is decomposed into decoupled subproblems that are assigned to the microgrids. The decomposition of the network into a group of interconnected microgrids is properly done with the objective of obtaining self-sufficient and efficient microgrids. Having self-sufficient microgrids is important not only for the purpose of decoupling the dispatch problem of the network but also for enabling self-healing feature with respect to network failures [5], [6].

To this end, we develop a distributed graph-based partitioning algorithm specifically designed for the considered problem. Similar to [7], the algorithm can be seen as a distributed variation of the Kernighen-Lin method. The algorithm is a distributed implementation of the refining step in the partitioning method proposed in [8]. Furthermore, we also employ a periodical partitioning scheme using the proposed algorithm in order to update the partition so that it is suitable with the conditions of both the renewable power generation and loads, which fluctuate periodically. After the network is partitioned, each microgrid solves a local optimization problem to compute its control inputs.

Compared to those methods based on distributed optimization algorithms [1]-[4], the proposed approach requires less intensive communication since the local controllers do not communicate among them at each time instant. Instead, they only exchange information in the repartitioning process. However, the computed control inputs might be suboptimal since the decentralized formulation of the dispatch problem shrinks the feasibility region by not allowing any power exchange between two neighboring microgrids.

In summary, the main contribution of this paper is the decentralized energy management strategy that consists in periodical repartitioning to obtain self-sufficient and efficient microgrids and decomposing the dispatch problem into decoupled local subproblems. The remainder of the paper is structured as follows. Section II provides the model of the power network and the formulation of the economic dispatch problem. Section III explains the decentralized scheme with the proposed periodical repartitioning procedure. Moreover, Section IV showcases the performance of the proposed 
method via numerical simulations based on a well-known case study. Finally, Section V concludes the paper by providing a summary and some remarks about future work.

\section{Notations}

The set of real numbers and integers are denoted by $\mathbb{R}$ and $\mathbb{Z}$, respectively. Moreover, for any $a \in \mathbb{R}, \mathbb{R}_{\geq a}$ denotes all real numbers in the set $\{b: b \geq a, b \in \mathbb{R}\}$. A similar definition can be used for $\mathbb{Z}_{\geq a}$ and the strict inequality case. For column vectors $v_{i}$ with $i \in \mathcal{L}=\left\{l_{1}, \ldots, l_{m}\right\}$, the operator $\left[v_{i}^{\top}\right]_{i \in \mathcal{L}}^{\top}$ denotes the column-wise concatenation, i.e., $\left[v_{i}^{\top}\right]_{i \in \mathcal{L}}^{\top}=\left[v_{l_{1}}^{\top}, \cdots, v_{l_{m}}^{\top}\right]^{\top}$. The set cardinality is denoted by $|\cdot|$ and the floor operator is denoted by $\lfloor\cdot\rfloor$. Finally, discrete-time instants are denoted by the subscript $k$.

\section{Problem Formulation}

\section{A. Model of the Network}

Let an electrical power network be described by an undirected graph $\mathcal{G}=(\mathcal{N}, \mathcal{E})$, where $\mathcal{N}=\{1,2, \ldots, n\}$ denotes the set of nodes that represents all busses in the network and $\mathcal{E} \subseteq \mathcal{N} \times \mathcal{N}$ denotes the set of edges (lines) that connect the busses. Additionally, denote the set of neighbors of bus $i$ by $\mathcal{N}_{i}$, i.e., $\mathcal{N}_{i}=\{j:(i, j) \in \mathcal{E}\}$. The components in the network are bus loads, which are uncertain, distributed generation units, which might be dispatchable or non-dispatchable, and energy storage devices. The model of each component type for an economic dispatch problem is described as follows:

a) Loads: The uncertain loads are modeled as follows:

$$
p_{i, k}^{1}=\hat{p}_{i, k}^{1}+w_{i, k}^{1}, \quad \forall i \in \mathcal{N},
$$

where $p_{i, k}^{1}, \hat{p}_{i}^{\mathrm{l}} \in \mathbb{R}_{\geq 0}$ denote the uncertain load of bus $i$ and its forecast, respectively, while $w_{i, k}^{\mathrm{l}} \in \mathbb{R}$ represent the disturbance/uncertainty of the load. Furthermore, we assume that the load forecast, $\hat{p}_{i}^{l}$, can be obtained and the disturbance, $w_{i, k}^{l}$, is bounded, i.e., $\left|w_{i, k}^{l}\right| \leq \bar{w}_{i}^{l}, \forall i \in \mathcal{N}$, where $\bar{w}_{i}^{l} \in \mathbb{R}_{\geq 0}$ denotes the bound of $w_{i, k}^{\mathrm{l}}$ that is known.

b) Distributed generation units: The power produced by non-dispatchable distributed generation units that are based on renewable energy sources, e.g., solar-based and wind-based power, is modeled similarly as uncertain loads. Denote the power produced by such generation units by $p_{i, k}^{\text {re }} \in \mathbb{R}_{\geq 0}$, its forecast by $\hat{p}_{i, k}^{\text {re }} \in \mathbb{R}_{\geq 0}$, its uncertainty by $w_{i, k}^{\mathrm{re}} \in \mathbb{R}$, and the bound of its uncertainty by $\bar{w}_{i}^{\mathrm{re}}$, i.e., $\left|w_{i, k}^{\mathrm{re}}\right| \leq \bar{w}_{i}^{\mathrm{re}}, \forall i \in \mathcal{N}^{\mathrm{re}}$, where $\mathcal{N}^{\mathrm{re}} \subset \mathcal{N}$ is the set of busses that have non-dispatchable renewable generation units. Similar to the load model, the renewable based power production is represented as follows:

$$
p_{i, k}^{\mathrm{re}}=\hat{p}_{i, k}^{\mathrm{re}}+w_{i, k}^{\mathrm{re}}, \forall i \in \mathcal{N}^{\mathrm{re}} .
$$

We also assume that the forecast and the bound, $\bar{w}_{i}^{\mathrm{re}}$, can be obtained. On the other hand, the dispatchable generators are modeled by production capacity constraints, as follows:

$$
0 \leq p_{i, k}^{\mathrm{g}} \leq \bar{p}_{i}^{\mathrm{g}}, \quad \forall i \in \mathcal{N}^{\mathrm{dg}},
$$

where $p_{i, k}^{\mathrm{g}}$ denotes the power produced by the dispatchable generator at bus $i, \bar{p}_{i, k}^{\mathrm{g}}$ denotes the maximum production capacity, and $\mathcal{N}^{\mathrm{dg}} \subset \mathcal{N}$ denotes the set of busses that have dispatchable generation units.

c) Energy storage devices: Denote the set of busses that have storage devices by $\mathcal{N}^{\text {st }} \subset \mathcal{N}$. The dynamics of a storage device at bus $i \in \mathcal{N}^{\text {st }}$ is represented as an integrator model, as follows:

$$
x_{i, k+1}=a_{i} x_{i, k}+b_{i} p_{i, k}^{\mathrm{st}},
$$

where $x_{i, k} \in \mathbb{R}_{\geq 0}$ denotes the state-of-charge (SoC) of the storage device, $p_{i, k}^{\text {st }} \in \mathbb{R}$ denotes the power delivered to/from the storage, $a_{i} \in(0,1]$ denotes the the efficiency of the storage and $b_{i}=-\frac{T_{\mathrm{s}}}{e_{\mathrm{cap}, i}}$, where $T_{\mathrm{s}}$ and $e_{\mathrm{cap}, i}$ denote the sampling time and the maximum capacity of the storage, respectively. Furthermore, the operational limits of the storage are represented by the following constraints:

$$
\begin{aligned}
& x_{i}^{\min } \leq x_{i, k} \leq x_{i}^{\max }, \\
& -p_{i}^{\mathrm{ch}} \leq p_{i, k}^{\mathrm{st}} \leq p_{i}^{\mathrm{dh}},
\end{aligned}
$$

where $x_{i}^{\min }, x_{i}^{\max } \in[0,1]$ denote the minimum and the maximum SoC of the storage of microgrid $i$, respectively. Moreover, $p_{i}^{\text {ch }} \in \mathbb{R}_{\geq 0}$ and $p_{i}^{\text {dh }} \in \mathbb{R}_{\geq 0}$ denote the maximum charging and discharging power of the storage.

Furthermore, each bus $i \in \mathcal{N}$ also has power balance equations, as follows:

$$
\begin{array}{r}
p_{i, k}^{\mathrm{re}}+p_{i, k}^{\mathrm{g}}+p_{i, k}^{\mathrm{st}}+p_{i, k}^{\mathrm{im}}+\sum_{j \in \mathcal{N}_{i}} p_{j i, k}^{\mathrm{t}}-p_{i, k}^{\mathrm{l}}=0, \\
p_{i j, k}^{\mathrm{t}}+p_{j i, k}^{\mathrm{t}}=0, \quad \forall j \in \mathcal{N}_{i},
\end{array}
$$

where $p_{i, k}^{\text {im }} \in \mathbb{R}_{\geq 0}$ denotes the power imported from the main grid if bus $i$ belongs to the set of busses that are connected to the main grid, denoted by $\mathcal{N}^{\mathrm{im}}$, and $p_{i j, k}^{\mathrm{t}} \in \mathbb{R}$ denotes the power transferred to/from the neighbor bus $j \in \mathcal{N}_{i}$. Equation (7) can be considered as local power balance whereas (8) couples two neighboring busses.

Now, we are in a position to state a robust economic dispatch problem of the network that is based on an MPC formulation. Firstly, in order to robustify the decisions, the worst case scenarios of the disturbances, i.e.,

$$
w_{i, k}^{\mathrm{l}}=\bar{w}_{i}^{\mathrm{l}}, \forall i \in \mathcal{N}, \quad w_{i, k}^{\mathrm{re}}=-\bar{w}_{i}^{\mathrm{re}}, \forall i \in \mathcal{N}^{\mathrm{re}},
$$

are taken into account. Secondly, define the vector of decision variables of each bus $i \in \mathcal{N}$ by $\boldsymbol{u}_{i, k}=$ $\left[\begin{array}{llll}p_{i, k}^{\mathrm{st}} & p_{i, k}^{\mathrm{g}} & p_{i, k}^{\mathrm{im}} & \boldsymbol{u}_{i, k}^{\mathrm{c} \top}\end{array}\right]^{\top} \in \mathbb{R}^{3+\left|\mathcal{N}_{i}\right|}$, where $\boldsymbol{u}_{i, k}^{\mathrm{c}}=$ $\left[p_{i j, k}^{\mathrm{t}}\right]_{i \in \mathcal{N}_{i}}$. In this regard, the following additional constraints must be imposed:

$$
\begin{aligned}
& p_{i, k}^{\mathrm{g}}=0, \forall i \notin \mathcal{N}^{\mathrm{dg}}, \quad p_{i, k}^{\mathrm{st}}=0, \forall i \notin \mathcal{N}^{\mathrm{st}}, \\
& p_{i, k}^{\mathrm{im}}=0, \forall i \notin \mathcal{N}^{\mathrm{im}}, \quad p_{i, k}^{\mathrm{re}}=0, \forall i \notin \mathcal{N}^{\mathrm{re}} .
\end{aligned}
$$

Thirdly, an economic quadratic cost function is considered as follows:

$$
J_{i, k}=\boldsymbol{u}_{i, k}^{\top} R_{i} \boldsymbol{u}_{i, k},
$$

where $\left.R_{i}=\operatorname{diag}\left(\left[\begin{array}{lll}c_{i}^{\mathrm{st}} & c_{i}^{\mathrm{g}} & c_{i}^{\mathrm{im}}\end{array} c_{i j}^{\mathrm{t}}\right]_{j \in \mathcal{N}_{i}}\right]\right) \succ 0$, in which $c_{i}^{\mathrm{st}}, c_{i}^{\mathrm{g}}, c_{i}^{\mathrm{im}}, c_{i j}^{\mathrm{t}} \in \mathbb{R}_{>0}$ denote the per-unit cost of storage operation, the per-unit cost of producing energy, the per-unit cost of buying energy from the main grid, and the per-unit 
cost of transferring energy to/from the neighbor due to losses [9]. Therefore, the optimization problem behind an MPCbased economic dispatch is stated as follows:

$$
\begin{gathered}
\underset{\left\{\left\{\boldsymbol{u}_{i, \ell \mid k}\right\}_{i \in \mathcal{N}\}_{\ell=k}^{k+h_{p}-1}}\right.}{\operatorname{minimimize}} \sum_{i \in \mathcal{N}} \sum_{\ell=k}^{k+h_{p}-1} J_{i, \ell}\left(\boldsymbol{u}_{i, \ell \mid k}\right) \\
\text { subject to } \boldsymbol{F}_{i} \boldsymbol{u}_{i, \ell \mid k} \leq \boldsymbol{f}_{i, \ell}, \forall i \in \mathcal{N}, \\
\boldsymbol{u}_{i, \ell \mid k}^{\mathrm{c}}+\sum_{j \in \mathcal{N}_{i}} \boldsymbol{G}_{i j} \boldsymbol{u}_{j, \ell \mid k}^{\mathrm{c}}=\mathbf{0}, \forall i \in \mathcal{N},
\end{gathered}
$$

for all $\ell \in\left\{k, \ldots, k+h_{p}-1\right\}$, where $h_{p} \in \mathbb{Z}_{\geq 1}$ denotes the prediction horizon. Note that the local constraints (12b) are constructed from (1)-(7), (9), and (10). The constraints of one node to another can be different depending on the components that one node has. Moreover, the coupled constraints (12c) are constructed from (8).

\section{B. Energy Management Based on Interconnected Microgrid Model}

A non-centralized control scheme is considered in this network. Suppose that the network is partitioned into $m$ subsystems, which are referred as microgrids. Then, $m$ local controllers are assigned to the subsystems and each of them will compute the decision variables associated to its subsystem. In this regard, Problem (12) is recasted as an economic dispatch problem of interconnected microgrids. Proposition 1 provides the reformulation of Problem (12) into an economic dispatch problem of an interconnectedmicrogrid system.

Definition 1: The set $\mathcal{M}=\left\{\mathcal{M}_{1}, \mathcal{M}_{2}, \ldots, \mathcal{M}_{m}\right\}$ defines $m$ non-overlapping partitions of graph $\mathcal{G}=(\mathcal{N}, \mathcal{E})$ if $\bigcup_{p=1}^{m} \mathcal{M}_{p}=\mathcal{N}$ and $\mathcal{M}_{p} \cap \mathcal{M}_{q}=\emptyset$, for any $\mathcal{M}_{p}, \mathcal{M}_{q} \in \mathcal{M}$ and $p \neq q$.

Proposition 1: Suppose that, at time instant $k$, the network is partitioned into $m$ non-overlapping microgrids, defined by the set $\mathcal{M}_{k}=\left\{\mathcal{M}_{1, k}, \mathcal{M}_{2, k}, \ldots, \mathcal{M}_{m, k}\right\}$ (Definition 1). Furthermore, denote the set of boundary busses of microgrid $\mathcal{M}_{p, k}$, i.e., busses that are connected (coupled) to at least one bus that belongs to another microgrid by $\mathcal{M}_{p, k}^{\mathrm{c}}=\{i$ : $\left.(i, j) \in \mathcal{E}, i \in \mathcal{M}_{p, k}, j \in \mathcal{N} \backslash \mathcal{M}_{p, k}\right\}$. Then, Problem (12) is equivalent to

$$
\begin{aligned}
& \underset{\left\{\left\{\boldsymbol{u}_{i, \ell \mid k}\right\}_{i \in \mathcal{N}}\right\}_{\ell=k}^{k+h_{p}-1}}{\operatorname{minimize}} \sum_{\ell=k}^{k+h_{p}-1} \sum_{p=1}^{m} J_{p, \ell}^{\mu} \\
& \text { subject to } \quad \boldsymbol{F}_{i} \boldsymbol{u}_{i, \ell \mid k} \leq \boldsymbol{f}_{i, \ell}, \forall i \in \mathcal{M}_{p, k} \text {, } \\
& \boldsymbol{u}_{i, \ell \mid k}^{\mathrm{c}}+\sum_{j \in \mathcal{N}_{i} \cap \mathcal{M}_{p, k}} \boldsymbol{G}_{i j} \boldsymbol{u}_{j, \ell \mid k}^{\mathrm{c}}=\mathbf{0}, \forall i \in \mathcal{M}_{p, k} \text {, } \\
& \boldsymbol{u}_{i, \ell \mid k}^{\mathrm{c}}+\sum_{j \in \mathcal{N}_{i} \backslash \mathcal{M}_{p, k}} \boldsymbol{G}_{i j} \boldsymbol{u}_{j, \ell \mid k}^{\mathrm{c}}=\mathbf{0}, \forall i \in \mathcal{M}_{p, k}^{\mathrm{c}},
\end{aligned}
$$

for all $\mathcal{M}_{p, k} \in \mathcal{M}_{k}$ and $\ell \in\left\{k, \ldots, k+h_{p}-1\right\}$, where $J_{p, \ell}^{\mu}=\sum_{i \in \mathcal{M}_{p, k}} J_{i, \ell}\left(\boldsymbol{u}_{i, \ell \mid k}\right)$.

Proof: The cost function (12a) is equal to (13a) and (12b) is equivalent to (13b) by the construction of $\mathcal{M}_{k}$. Furthermore, (12c) is decomposed into (13c) and (13d).
Remark 1: From the perspective of microgrids, (13b) and (13c) are local constraints whereas (13d) are coupled constraints.

One challenge to solve (13) in a non-centralized scheme is the existence of the coupled constraints (13d). In Section III, a decentralized method to solve (13) is proposed. The method is a combination of implementing a periodical partitioning algorithm, with the objective of obtaining self-sufficient and efficient interconnected microgrids in order to decompose (13) and solving decoupled economic dispatch problems, derived from the decomposition of (13).

\section{PRoposed Method}

\section{A. Repartitioning Algorithm}

Since the loads and the renewable power generation vary and might differ a lot from one period to another, we propose to repartition the network periodically to maintain self-sufficiency, which might be degraded due to the changes of the load and generation profiles. Furthermore, despite the uncertainties, one can observe periodical patterns of the renewable power generation and the loads, e.g., as reported in [10]. Hence, let the periodicity of the repartitioning process be denoted by $\tau \in \mathbb{Z}_{\geq 1}$. This fact implies that the network is repartitioned at $\bar{k}=\tau, 2 \tau, 3 \tau, \ldots$, which are called the repartitioning instants, and $\mathcal{M}_{k}=\mathcal{M}_{\lfloor k / \tau\rfloor \tau}$, for any $k \in \mathbb{Z}_{\geq 0}$. Furthermore, suppose that the network has initially been partitioned into $m$ non-overlapping microgrids and denote the set of initial partition at $k=0$ by $\mathcal{M}_{0}=\left\{\mathcal{M}_{1,0}, \mathcal{M}_{2,0}, \ldots, \mathcal{M}_{m, 0}\right\}$. In order to state the repartitioning problem, first we define the local cost function for each microgrid $\mathcal{M}_{p, k}$, denoted by $J^{\pi}\left(\mathcal{M}_{p, k}\right)$ and consists of two components, as follows:

$$
J^{\pi}\left(\mathcal{M}_{p, k}\right)=\alpha J_{p, k}^{\mathrm{im}}+J_{p, k}^{\mathrm{ef}},
$$

where $J_{p, k}^{\mathrm{im}}$ denotes the imbalance cost and $J_{p, k}^{\mathrm{ef}}$ denotes the efficiency cost, whereas $\alpha$ denotes a positive weight and can be regarded as a tuning parameter of the repartitioning procedure. The imbalance cost is defined as follows:

$$
J_{p, k}^{\mathrm{im}}=\sum_{\ell=k}^{k+\tau-1} \max \left(0, \sum_{i \in \mathcal{M}_{p, k}} p_{i, \ell}^{\mathrm{li}}\right),
$$

where $p_{i, \ell}^{\mathrm{li}}$, which denotes the local power imbalance, is expressed as

$$
p_{i, \ell}^{\mathrm{li}}=-\bar{p}_{i}^{\mathrm{g}}-p_{i, \ell}^{\mathrm{re}}+p_{i, \ell}^{\mathrm{l}},
$$

in which $p_{i, \ell}^{\mathrm{re}}$ and $p_{i, \ell}^{\mathrm{l}}$ follow (1) and (2), respectively, and the worst case disturbances, which are shown in (9), are considered to robustify the solution against the uncertainties. The imbalance cost (15) penalizes a microgrid that does not have enough local power resource to meet the loads. Moreover, the efficiency cost is defined as follows:

$$
\begin{array}{r}
J_{p, k}^{\mathrm{ef}}=\min _{\left\{\left\{\boldsymbol{u}_{i, \ell \mid k}\right\}_{i \in \mathcal{M}_{p, k}}\right\}_{\ell=k}^{k+\tau+h_{p}-1}} \sum_{\ell=k}^{k+\tau+h_{p}-1}\left(J_{p, \ell}^{\mu}+J_{p, \ell}^{\epsilon}\right) \\
\text { s. t. (13b)-(13c), } \forall \ell \in\left\{k, \ldots, k+\tau+h_{p}-1\right\},
\end{array}
$$


where $J_{p, \ell}^{\epsilon}$ adds extra cost on the power transferred between one microgrid to another and is defined as $J_{p, \ell}^{\epsilon}=\sum_{i \in \mathcal{M}_{p, k}^{\mathrm{c}}} \sum_{j \in \mathcal{N}_{i} \backslash \mathcal{M}_{p, k}} c_{i}^{\text {et }}\left(p_{i j, \ell}^{\mathrm{t}}\right)^{2}$, where $c_{i}^{\text {et }}$ is the extra per-unit cost of transferring power and can be set quite large to minimize the usage of transferred power. As can be seen in (17), in order to compute $J_{p, k}^{\text {ef }}$, the local controller must solve a local economic dispatch problem derived from (13) over $\tau+h_{p}$ time instants.

Thus, for each repartitioning instant, $k=\tau, 2 \tau, 3 \tau, \ldots$, the repartitioning problem that must be solved is stated as follows:

$$
\underset{\mathcal{M}_{k}}{\operatorname{minimize}} \sum_{p=1}^{m} J^{\pi}\left(\mathcal{M}_{p, k}\right)
$$

subject to

$$
\begin{aligned}
& \bigcup_{p=1}^{m} \mathcal{M}_{p, k}=\mathcal{N}, \\
& \mathcal{M}_{p, k} \cap \mathcal{M}_{q, k}=\emptyset, \forall \mathcal{M}_{p, k}, \mathcal{M}_{q, k} \in \mathcal{M}_{k}, p \neq q, \\
& \lambda_{2}\left(L\left(\mathcal{G}_{p, k}\right)\right)>0, \quad \forall \mathcal{M}_{p, k} \in \mathcal{M}_{k}, \\
& \mathcal{M}_{k}^{(0)}=\mathcal{M}_{k-1},
\end{aligned}
$$

where $\mathcal{G}_{p, k}=\left(\mathcal{M}_{p, k}, \mathcal{E}_{p, k}\right)$ denotes the subgraph of microgrid $p$, with the set of edges denoted by $\mathcal{E}_{p, k}=\{(i, j) \in$ $\left.\mathcal{E}: i, j \in \mathcal{M}_{p, k}\right\}$ and $\lambda_{2}\left(L\left(\mathcal{G}_{p, k}\right)\right)$ denotes the second smallest eigenvalue of the Laplacian matrix of subgraph $\mathcal{G}_{p, k}$. Equation (18d) implies the requirement of having connected microgrids, i.e., the subgraph formed by each microgrid is connected. This constraint is imposed to avoid decoupling within each microgrid. Furthermore, $\mathcal{M}_{k}^{(0)}$ denotes the initial partition, which is obtained from the partition at the previous time instant, $k-1$. In particular, for $k=\tau, \mathcal{M}_{k}^{(0)}=\mathcal{M}_{0}$. Note that Assumption 1, which is related to the initial partition $\mathcal{M}_{0}$, is considered.

Assumption 1: The initial partition $\mathcal{M}_{0}$ is nonoverlapping with connected microgrids.

Remark 2: The partition $\mathcal{M}_{0}$ can be obtained by solving an optimal microgrid construction problem [5], [6].

To solve the repartitioning problem while taking into account the setup of the system, an iterative local improvement algorithm that is performed in a distributed and synchronous manner is proposed. The main idea of the algorithm is, at each iteration, one node (bus) is proposed to be moved from one microgrid to a neighboring microgrid in order to improve the total cost. Denote the iteration number by superscript $(r)$ and consider the initial partition $\mathcal{M}_{k}^{(0)}$. At the $r^{\text {th }}$ iteration, suppose that microgrid $p$ is chosen to propose a node that will be moved. Then, the steps at each iteration are described below.

\section{Algorithm 1: Repartitioning Procedure}

1) Microgrid $p$ computes $J^{\pi}\left(\mathcal{M}_{p, k}^{(r)}\right)$, which is the local cost function at the $r^{\text {th }}$ iteration, based on (14).

2) Microgrid $p$ computes a node that will be offered to be moved, denoted by $\theta_{p}$ as follows:

$$
\theta_{p} \in \arg \min _{\theta \in \mathcal{M}_{p, k}^{\theta(r)}} J^{\pi}\left(\mathcal{M}_{p, k}^{(r)} \backslash\{\theta\}\right),
$$

where $\mathcal{M}_{p, k}^{\theta(r)} \subseteq \mathcal{M}_{p, k}^{\mathrm{c}(r)}$ is a subset of boundary busses that do not disconnect microgrid $p$ when removed, i.e., $\mathcal{M}_{p, k}^{(r)} \backslash\{\theta\}$, for $\theta \in \mathcal{M}_{p, k}^{\theta(r)}$, is connected. The node $\theta_{p}$ is randomly selected from the set of minimizers.

3) Microgrid $p$ computes the local cost difference if $\theta_{p}$ is moved out of microgrid $p$, i.e.,

$$
\Delta J_{p}^{\pi(r)}=J^{\pi}\left(\mathcal{M}_{p, k}^{(r)} \backslash\left\{\theta_{p}\right\}\right)-J^{\pi}\left(\mathcal{M}_{p, k}^{(r)}\right) .
$$

4) Microgrid $p$ shares the information of $\theta_{p}$ and $\Delta J_{p}^{\pi(r)}$ to the related neighboring microgrids $\mathcal{M}_{q, k}^{(r)} \in \mathcal{N}_{\theta_{p}}^{\prime}=$ $\left\{\mathcal{M}_{q, k}^{(r)}:\left(\theta_{p}, j\right) \in \mathcal{E}, j \in \mathcal{M}_{q, k}^{(r)}\right\}$.

5) All neighbors $\mathcal{M}_{q, k}^{(r)} \in \mathcal{N}_{\theta_{p}}^{\prime}$ compute the expected total cost difference if $\theta_{p}$ is moved from microgrid $\mathcal{M}_{p, k}^{(r)}$ to microgrid $\mathcal{M}_{q, k}^{(r)}$, as follows:

$$
\Delta J_{\mathrm{t}, q}^{\pi(r)}=J^{\pi}\left(\mathcal{M}_{q, k}^{(r)} \cup\left\{\theta_{p}\right\}\right)-J^{\pi}\left(\mathcal{M}_{q, k}^{(r)}\right)+\Delta J_{p}^{\pi(r)},
$$

and send the information of $\Delta J_{\mathrm{t}, q}^{\pi(r)}$ to microgrid $p$.

6) Microgrid $p$ selects the neighbor that will receive $\theta_{p}$, as follows: $q^{\star} \in \arg \min _{q \in \mathcal{N}_{\theta_{p}}^{\prime}} \Delta J_{\mathrm{t}, q}^{\pi(r)}$, where $q^{\star}$ is randomly chosen from the set of minimizers.

7) If $\Delta J_{\mathrm{t}, q^{\star}}^{\pi(r)} \leq 0$, then the partition is updated as follows: $\mathcal{M}_{p, k}^{(r+1)}=\mathcal{M}_{p, k}^{(r)} \backslash\left\{\theta_{p}\right\}, \quad \mathcal{M}_{q^{\star}, k}^{(r+1)}=\mathcal{M}_{q^{\star}, k}^{(r)} \cup\left\{\theta_{p}\right\}$. Otherwise, the algorithm jumps to the next iteration, $r+1$.

Note that, the number of repartitioning iterations is upperbounded by a constant, denoted by $\bar{r}$. In addition, Proposition 2 characterizes the solution obtained by the proposed algorithm.

Proposition 2: Let Assumption 1 hold. The solution of Algorithm 1 at any repartitioning instant, $k=\tau, 2 \tau, 3 \tau, \ldots$, is a non-overlapping partition with connected microgrids and converges to a local minimum.

\section{B. Decentralized MPC for Economic Dispatch}

Based on the partition obtained in Section III-A, each microgrid $\mathcal{M}_{p, k} \in \mathcal{M}_{k}$ computes its local decision variables for the economic dispatch problem in a decentralized MPC framework at each time instant. In order to describe the method, first we classify the microgrids in the network based on Definition 2.

Definition 2: A microgrid $\mathcal{M}_{p, k} \in \mathcal{M}_{k}$ is self-sufficient at time instant $k$ if the imbalance cost (15) at the last repartitioning instant is zero, i.e., $J_{p,\lfloor k / \tau\rfloor \tau}^{\mathrm{im}}=0$.

Suppose that all microgrids in the network are selfsufficient. Then, each $\mathcal{M}_{p, k} \in \mathcal{M}_{k}$ solves a local economic dispatch problem at each $k$, as follows:

$$
\begin{aligned}
& \underset{\left\{\left\{\boldsymbol{u}_{i, \ell \mid k}\right\}_{\left.i \in \mathcal{M}_{p, k}\right\}_{\ell=k}^{k+h_{p}-1}}\right.}{\operatorname{minimize}} \sum_{\ell=k}^{k+h_{p}-1} J_{p, \ell}^{\mu} \\
& \text { subject to (13b)-(13d), } \\
& \boldsymbol{u}_{j, \ell \mid k}^{\mathrm{c}}=\mathbf{0}, \forall j \in \mathcal{N}_{i} \backslash \mathcal{M}_{p, k}, \forall i \in \mathcal{M}_{p, k}^{\mathrm{c}},
\end{aligned}
$$

for all $\ell \in\{k, \ldots, k+\tau-1\}$. In this setup, each microgrid does not allow the transfer of power to/from its neighbors. 
Hence, we manage to decompose (13) into $m$ decoupled subproblems, which can be solved without requiring communication among the microgrids.

Now, we discuss the case in which there exists a set of microgrids that are not self-sufficient. For this case, the main idea is to let the self-sufficient microgrids to help. To this end, the microgrids that are not self-sufficient solve a relaxed version of Problem (19), which is similar to the problem solved to compute $J_{p, k}^{\mathrm{ef}}$, i.e.,

$$
\begin{aligned}
& \underset{\left\{\left\{\boldsymbol{u}_{i, \ell \mid k}\right\}_{\left.i \in \mathcal{M}_{p, k}\right\}_{\ell=k}^{k+h_{p}-1}}\right.}{\operatorname{minimize}} \sum_{\ell=k}^{k+h_{p}-1}\left(J_{p, \ell}^{\mu}+J_{p, \ell}^{\epsilon}\right) \\
& \text { subject to (13b)-(13c), } \forall \ell \in\left\{k, \ldots, k+h_{p}-1\right\} \text {. }
\end{aligned}
$$

Therefore, it is regarded that the insufficiency can be met by transferring power from the neighbors if necessary. Notice that extra cost is given to the power transfer to/from neighboring microgrid by $J_{p, \ell}^{\epsilon}$. Furthermore, each microgrid has a storage unit that can help to deal with self-sufficiency issue by storing some excess of energy at one particular time instant and delivering this energy back to the grid when needed. Nevertheless, when the energy from neighboring microgrids is needed, then it might lead to the violation of the coupling constraints (8) for some of the boundary busses. The violation of these constraints implies imbalances in the network. We assume that there exist low-level controllers of the generators that handle the imbalances [11], implying the suboptimality of the operation. A proposal to combine decentralized and distributed schemes to tackle this issue in the dispatch level is currently being investigated. The main idea of the proposal is that a microgrid that is not selfsufficient employs a distributed MPC method with some of its neighbors so that they can cooperate to meet their loads efficiently whereas a neighborhood of self-sufficient microgrids can still perform the decentralized dispatch.

\section{The Overall Method}

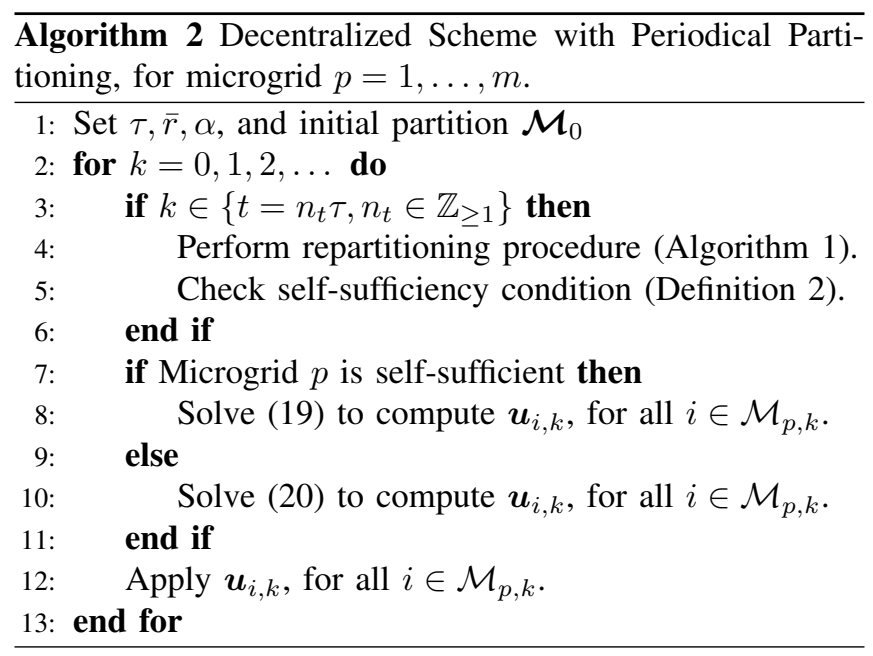

The proposed scheme is summarized in Algorithm 2. Since at each time instant the decision variables are computed in a decentralized manner, the local controllers do not need to exchange information among each other as in typical distributed optimization algorithms. Note that iterative distributed optimization algorithms usually require the exchanging of information at each iteration and this can introduce additional issues such as information delay, communication failures, and cyber attacks [12], [13]. On the other hand, the computed decision variables might be suboptimal since the solution space is limited by (19b). In this regard, performing the periodical repartitioning procedure reduces the suboptimality since it takes into account the economical efficiency as one of the partitioning criteria.

\section{CAse Study}

The case study considered is based on the PG\&E 69-bus distribution network, as shown in Fig. 1. Dispatchable, solarbased distributed generation, and storage units are added to the network. Furthermore, the parameters of the network components are given in Table I. The load profiles and forecasts are generated based on the available load data, which are regarded as the maximum loads, and based on the typical residential and commercial load profiles. The busses that have maximum load greater than $100 \mathrm{~kW}$ are considered to have a commercial load profile. Otherwise, they have a residential load profile.

The partition obtained in [6] and depicted in Fig. 1.a. is used as the initial and default partition. Note that the default partition is set to be self-sufficient and the goal of the numerical simulations is to show the performance improvement obtained by applying periodical partitioning for the decentralized scheme. Therefore, three simulation scenarios are considered, as stated in Table II. In scenario 1, the centralized MPC scheme, i.e., solving (13) at each time instant in a centralized manner, is applied. In scenario 2, the proposed decentralized scheme with periodical repartitioning is applied with $\tau=16$ steps, $\bar{r}=80$, and $\alpha=10^{4}$. Note that the network is repartitioned every 16 steps based on the periodicity of the uncertainty level that is observed. Whereas, in scenario 3, the decentralized scheme is applied without the repartitioning procedure, i.e., the default partition (Fig. 1.a.) is kept constant for the whole simulation. For each scenario, the sampling time is 15 minutes and the simulation time is one day.

Table II shows that, by applying periodical partitioning (scenario 2), the performance of the whole network is better than that of scenario 3, as expected. Furthermore, the performance in scenario 2 is suboptimal compared to that of scenario 1. Note that the optimal performance (scenario 1) requires exchanging power among the microgrids, explaining the suboptimality of the system in scenarios 2 and 3 , where exchanging power among microgrids is not allowed. In addition, Fig. 1 also shows how the partition changes throughout the simulation in scenario 2. Note that even though the repartitioning process happens with the period of $\tau=16$ time steps, at some repartitioning instants, i.e., $k=32,48$, and 80 , the partition is kept the same since it is assessed that changing the partition would not improve or maintain the performance of the system. 
TABLE I

PARAMETERS OF THE NeTWORK COMPONENTS

\begin{tabular}{cccc}
\hline Parameters & Value & Unit & Bus \\
\hline$p_{i}^{\mathrm{g}, \text { min }}, p_{i}^{\mathrm{g}, \text { max }}$ & 0,500 & $\mathrm{~kW}$ & $i \in \mathcal{N}^{\mathrm{dg}}$ \\
$x_{i}^{\mathrm{min}}, x_{i}^{\mathrm{max}}, x_{i, 0}$ & $30,80,50$ & $\%$ & $i \in \mathcal{N}^{\text {st }}$ \\
$p_{i}^{\mathrm{ch}}, p_{i}^{\mathrm{dh}}$ & 200,200 & $\mathrm{~kW}$ & $i \in \mathcal{N}^{\text {st }}$ \\
$e_{\text {cap }, i}$ & 1000 & $\mathrm{kWh}$ & $i \in \mathcal{N}^{\text {st }}$ \\
$a_{i}$ & 0.98 & - & $i \in \mathcal{N}^{\text {st }}$ \\
$c_{i}^{\mathrm{st}}, c_{i}^{\mathrm{g}}$ & 1,10 & - & $i \in \mathcal{N}^{\mathrm{m}}$ \\
$c_{i}^{\mathrm{im}}, c_{i}^{\mathrm{t}}$ & 10,1 & - & $i \in \mathcal{N}^{-}$ \\
\hline
\end{tabular}

TABLE II

TOTAL Cost OF THE SYSTEM

\begin{tabular}{clc}
\hline Scenario & MPC Scheme & $\begin{array}{c}\text { Cost } \\
\text { (Proportional) }\end{array}$ \\
\hline 1 & Centralized & 1.00 \\
2 & $\begin{array}{l}\text { Decentralized with } \\
\text { Repartitioning }\end{array}$ & 1.12 \\
3 & $\begin{array}{l}\text { Decentralized without } \\
\text { Repartitioning }\end{array}$ & 1.20 \\
\hline
\end{tabular}

\section{CONCLusion And Future Work}

A decentralized MPC scheme that uses a periodical repartitioning method has been proposed to solve dynamic economic dispatch problem of power networks. The objective of the repartitioning method is to maintain self-sufficiency and efficiency of each microgrid. As future work, stochastic approaches are considered to relax the assumption of bounded uncertainty. Furthermore, a combination of distributed and decentralized control schemes is currently investigated to deal with the issues when some microgrids are not selfsufficient, as discussed in Section III-B. Moreover, further analysis of the proposed scheme, such as a suboptimality certificate, is also a part of the ongoing work.

\section{REFERENCES}

[1] G. K. H. Larsen, N. D. van Foreest, and J. M. A. Scherpen, "Distributed MPC applied to a network of households with micro-CHP and heat storage," IEEE Trans. Smart Grid, vol. 5, no. 4, pp. 21062114, 2014.

[2] T. Wang, D. O'Neill, and H. Kamath, "Dynamic control and optimization of distributed energy resources in a microgrid," IEEE Trans. Smart Grid, vol. 6, no. 6, pp. 2884-2894, 2015.

[3] K. Baker, J. Guo, G. Hug, and X. Li, "Distributed MPC for efficient coordination of storage and renewable energy sources across control areas," IEEE Trans. Smart Grid, vol. 7, no. 2, pp. 992-1001, 2016.

[4] P. Braun, L. Grüne, C. M. Kellett, S. R. Weller, and K. Worthmann, "A distributed optimization algorithm for the predictive control of smart grids," IEEE Trans. on Automat. Contr., vol. 61, no. 12, pp. 38983911, 2016.

[5] M. Barani, J. Aghaei, M. A. Akbari, T. Niknam, H. Farahmand, and M. Korps, "Optimal partitioning of smart distribution systems into supply-sufficient microgrids," IEEE Trans. Smart Grid, 2018, in press.

[6] S. A. Arefifar, Y. A. R. I. Mohamed, and T. H. M. El-Fouly, "Supply-adequacy-based optimal construction of microgrids in smart distribution systems," IEEE Trans. Smart Grid, vol. 3, no. 3, pp. 1491$1502,2012$.

[7] J. Barreiro-Gomez, C. Ocampo-Martinez, and N. Quijano, "Timevarying partitioning for predictive control design: Density-games approach," Journal of Process Control, vol. 75, pp. 1-14, 2019.

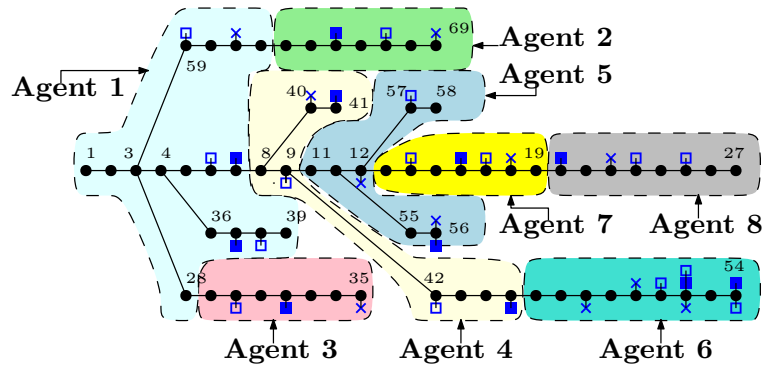

(a.)

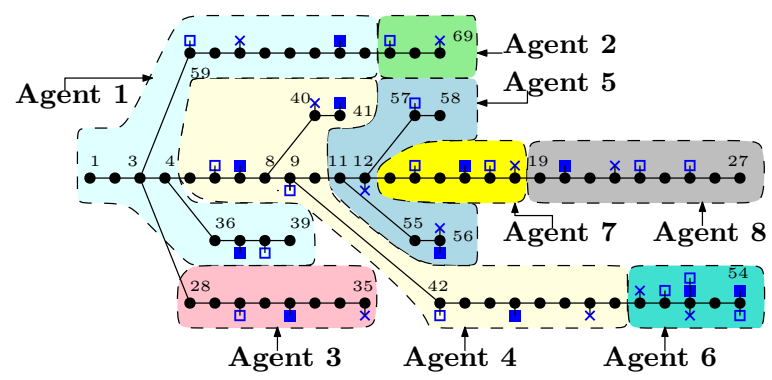

(b.)

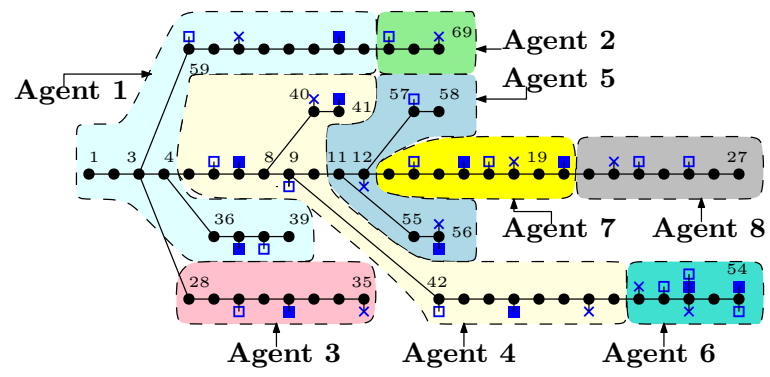

(c.)

Fig. 1. The topology of the PG\&E 69-bus distribution system. Squares indicate the distributed generation units, i.e., filled squares, $\mathbf{\square}$, and empty squares, $\square$, represent renewable generation units and a dispatchabale generators, respectively, whereas crosses, $\times$, indicate the storages. (a.) The 8-agent initial partition [6], for $k \in\{0,1, \ldots, 15\}$. (b.) The partition for $k \in\{16,17, \ldots, 63\}$. (c) The partition for $k \in\{64,65, \ldots, 95\}$.

[8] W. Ananduta, T. Pippia, C. Ocampo-Martinez, J. Sijs, and B. De Schutter, "Online partitioning method for decentralized control of linear switching large-scale systems," Journal of the Franklin Institute, 2019. [Online]. Available: https://doi.org/10.1016/j.jfranklin.2018.10.038

[9] C. A. Hans, P. Braun, J. Raisch, L. Grune, and C. Reincke-Collon, "Hierarchical distributed model predictive control of interconnected microgrids," IEEE Trans. Sustainable Energy, vol. 10, no. 1, pp. 407416, 2019.

[10] J. A. Jardini, C. M. V. Tahan, M. R. Gouvea, S. U. Ahn, and F. M. Figueiredo, "Daily load profiles for residential, commercial and industrial low voltage consumers," IEEE Trans. Power Delivery, vol. 15 , no. 1 , pp. 375-380, 2000.

[11] T. Morstyn, B. Hredzak, and V. G. Agelidis, "Control strategies for microgrids with distributed energy storage systems: An overview," IEEE Trans. Smart Grid, vol. 9, no. 4, pp. 3652-3666, 2018.

[12] X. Ge, F. Yang, and Q.-L. Han, "Distributed networked control systems: A brief overview," Information Sciences, vol. 380, pp. 117131, 2017.

[13] W. Ananduta, J. M. Maestre, C. Ocampo-Martinez, and H. Ishii, "Resilient distributed energy management for systems of interconnected microgrids," in Proceedings of the IEEE 57th Conference on Decision and Control (CDC), Miami, USA, 2018, pp. 3159-3164. 